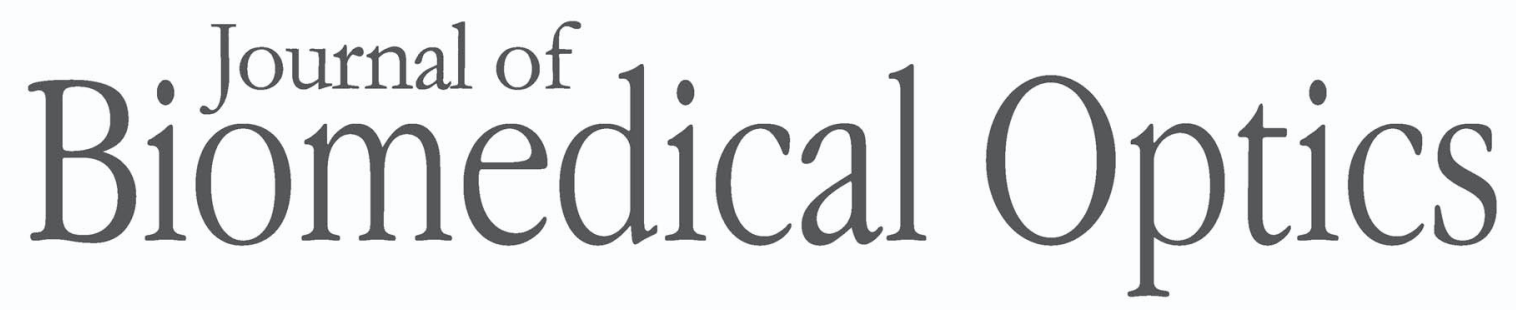

\title{
Optical delivery of liposome encapsulated chemical stimuli to neuronal cells
}

Giulietta Pinato

Tiziano Raffaelli

Elisa D'Este

Federica Tavano

Dan Cojoc 


\title{
Optical delivery of liposome encapsulated chemical stimuli to neuronal cells
}

\author{
Giulietta Pinato, Tiziano Raffaelli, Elisa D'Este, Federica Tavano, and Dan Cojoc \\ CNR, Istituto Officina dei Materiali, Laboratorio TASC, Ed. MM, Area Science Park- Basovizza, SS 14, \\ Km 163.5, 34149, Trieste, Italy
}

\begin{abstract}
Spatially confined and precise time delivery of neuroactive molecules is an important issue in neurophysiology. In this work we developed a technique for delivering chemical stimuli to cultured neurons consisting in encapsulating the molecules of interest in liposomes. These vectors were then loaded in reservoirs consisting of glass capillaries. The reservoirs were placed in the recording chamber and single liposomes were trapped and transported out by optical tweezers to the site of stimulation on cultured neurons. Finally, the release of liposome content was induced by application of UV-pulses, breaking the liposome membrane. The efficiency of encapsulation and release were first evaluated by loading the liposomes with fluorescein. In order to test the effect of the $\mathrm{UV}$-induced release, liposomes with diameter ranging from 1 to $10 \mu \mathrm{m}$ (fL to $\mathrm{pL}$ volumes), were filled with $\mathrm{KCl}$ and tested on neuronal cells. Neuronal cultures, loaded with $\mathrm{Ca}^{2+}$ dye, were monitored by imaging intracellular $\mathrm{Ca}^{2+}$. An efficient release from the liposomes was demonstrated by detectable calcium signals, indicating stimulated depolarization of the neuronal cells by $\mathrm{KCl}$. The present technique represents an alternative method for focal chemical stimulation of cultured cells that circumvents some of the limitations of microejection and photorelease of caged compounds. O 2011 Society of Photo-Optical Instrumentation Engineers (SPIE). [DOI: 10.1117/1.3616133]
\end{abstract}

Keywords: optical tweezers; neuron; neurotransmitter; release; laser; liposome.

Paper 11170RR received Apr. 7, 2011; revised manuscript received Jul. 2, 2011; accepted for publication Jul. 7, 2011; published online Sep. 1, 2011.

\section{Introduction}

The study of the synaptic function in the nervous system, including synaptic integration, development, and plasticity, and the investigation of fast desensitizing receptor kinetics are very often limited by the impossibility of properly mimicking the process of chemical release occurring in neuronal cells. ${ }^{1}$ Physiological chemical stimuli (release of neurotransmitters, neuromodulators, or neurotrophins) are characterized by fast release of small volumes, or even single molecules in spatially confined areas. The delivery of volumes in the range of femtoliters with sub-micron spatial precision would be therefore very useful, and would also provide a way to explore amplification mechanisms underlying metabotropic cascades by measuring cell global responses to the binding of single molecules.

Different techniques, that allow the stimulation of specific neuronal compartments (axons, somata, dendrites, and spines), have been developed with the aim of releasing chemical compounds noninvasively at sub-micron spatial resolution, with millisecond time precision and tight control of the volume released. Conventional methods for focal chemical delivery include puffers and microejectors ${ }^{2,3}$ that allow delivering nanoliters to picoliters of most of soluble drugs from glass micropipettes by pressure pulses. However, microejection techniques do not allow strict control of volumes released and present the risk of leakage from the micropipette. Moreover, ejection under pressure pulses produces spillover of the solution over large areas and can induce mechanical disturbance. The kinetics of

Address all correspondence to: Giulietta Pinato, CNR, Istituto Officina dei Materiali, Laboratorio TASC, Ed. MM, Area Science Park- Basovizza, SS 14, Km 163.5, 34149, Trieste, Italy; Tel: + 3904037584 17; Fax: + 390402267 67; E-mail: pinato@tasc.infm.it. delivery in these conditions definitely differs from physiological conditions. All in all, though technically straightforward, these methods do not appear suitable for single synapse stimulation or measurements of fast desensitizing receptor currents.

In order to obtain spatially restricted and faster stimulation, the method of photoactivation of caged compounds has been more recently developed becoming the most popular tool for focal stimulation of single synaptic sites. ${ }^{4,5}$ Photoactivation of caged compounds has the main advantage to be applicable for both extracellular and intracellular stimulation. Moreover, the temporal resolution of delivery is very high, ranging from submicroseconds to milliseconds and the location of the release is restricted to the area of incidence of the uncaging light. ${ }^{6}$

However, most of the caged compounds turned out to produce undesired side effects, being able to block both glycine and gamma-aminobutyric acid receptors ${ }^{7}$ and, up to now, there is no practical availability of caging of most of neuroactive molecules, precluding a large number of applications.

Recent methods circumvent the intrinsic limitations of the caging synthesis encapsulating molecules in micro/nanosources and liposomes. ${ }^{8,9}$ High time and spatial precision of delivery can be reached using these vectors in combination with optical manipulation and photolysis. In particular, Sun and $\mathrm{Chiu}^{9}$ demonstrated a method to stimulate CHO-M1 cells with carbachol molecules encapsulated in liposomes and released by membrane photolysis. Here, we present a development of this technique consisting in local delivery of $\mathrm{KCl}$ from single liposomes to stimulate neuronal cells in culture. We show that neuronal activity at a single cell level can be evoked using this technique. Another advantage is the combination of a

1083-3668/2011/16(9)/095001/5/\$25.00 (C) 2011 SPIE 
straightforward preparation with high spatial and temporal precision of delivery to neurons.

For the preparation of liposomes, phospholipids were dissolved at the concentration of $10 \mathrm{mg} / \mathrm{mL}$ in a solvent composed of chloroform:methanol $(2: 1, \mathrm{v} / \mathrm{v})$. The phospholipid composition was the following: Cholesterol: $9 \mu \mathrm{mol}, \mathrm{L}-\alpha$ Phosphatidylcholine: $63 \mu \mathrm{mol}$, Stearylamine: $18 \mu \mathrm{mol}$ (SigmaAldrich, cat. N. L4395). Liposomes were obtained using the lipid film hydration method. ${ }^{10}$ Aliquots of the phospholipid solution were dried in vacuum overnight. The obtained lipid films were finally hydrated with the solution to be encapsulated $(\mathrm{KCl}$ $100 \mathrm{mM}$, Sucrose $100 \mathrm{mM}$ ). After a brief incubation, liposomes were formed. Liposomes were then gently centrifuged $(5000 \mathrm{rpm} \times 3 \mathrm{~min})$ and rinsed three times with an isotonic solution [physiological buffered saline, (PBS)]. Sucrose $100 \mathrm{mM}$ was added to the hydration solution to improve liposome wash and to favor the trapping of large liposomes as previously reported. ${ }^{11}$ The size distribution of the liposomes was $6.30 \pm 4.04 \mu \mathrm{m}$. We could observe the presence of both multi- and mono/oligo-lamellar vesicles, based on qualitative optical criteria, i.e., shape and rim size. The stability was tested by filling the liposomes with fluorescein and maintained for days. Liposomes were finally charged in rectangular capillaries (Vitrotubes, \#5005, VitroCom, New Jersey) and subsequently transferred to the recording chamber. This allows selection of a single liposome from the capillary and positioning it at the site of interest. Considering the very low permeability $\left(10^{-11} \mathrm{~cm} / \mathrm{s}\right)($ Ref. 12$)$ of the $\mathrm{K}^{+}$cations across the phospholipid membranes at room temperature, which is well above the melting phase transition temperature of phosphatidylcholine, we can assume that the encapsulated aqueous solution has the same concentration as the rehydrated one and leakage of $\mathrm{K}^{+}$ ions is negligible. Moreover, after hydration in $\mathrm{KCl}$ solution, the liposomes were washed and quickly used in experiments, so that leakage of $\mathrm{K}^{+}$cations through the lipid bilayer could be definitely excluded. Preparation of $\left(\mathrm{Ca}^{2+}\right)$ ions encapsulated in liposomes has been previously reported ${ }^{13}$ and the ion concentration of the encapsulated solution was estimated to be the same as that of the rehydration solution in bulk.

Although a quantitative method for the evaluation of the encapsulation efficiency was proposed using photolysis and confocal microscopy of individual liposomes,${ }^{14}$ this technique allows only measurement of fluorescent molecules that was not the case of $\mathrm{KCl}$.

Cell cultures were obtained from hippocampi dissected from $\mathrm{P} 0-\mathrm{P} 1$ rats, in accordance with the regulation of the Italian Animal Welfare Act, and under the approval of a local veterinary service. Cells were plated at the concentration of $10^{5}$ cells $/ \mathrm{mL}$ on glass bottom petri-dishes, coated with polyornithine and $2 \%$ Matrigel (BD Biosciences). Neuronal cultures were maintained in an incubator at $5 \% \mathrm{CO}_{2}, 95 \%$ moisture, and $37^{\circ} \mathrm{C}$ in minimum essential medium with Earle's salts and Glutamax I (MEM, Life Technologies, Invitrogen) with 10\% FBS (Gibco), 35 mM D-glucose (Lancaster), $14 \mathrm{mM}$ Hepes (Sigma), $1 \mathrm{mM}$ vitamin B12 (Sigma), $0.36 \mathrm{mM}$ d-Biotin (Sigma), $30 \mu \mathrm{g} / \mathrm{mL}$ insulin (Sigma), $100 \mu \mathrm{g} / \mathrm{mL}$ bovine transferrin (Sigma), and antibiotics (Euroclone). The medium was changed every two days. Cultures were used after five to seven days in vitro.

Single liposomes were optically trapped and transported out of the reservoir to desired sites in the proximity of the cultured neurons by means of a custom IR optical tweezers implemented on an inverted microscope Nikon Eclipse TE-2000-E. The collimated $\mathrm{TEM}_{00}$ beam from a $1064 \mathrm{~nm}$ continuous wave $\mathrm{Yb}$ fiber laser (YLM-5, IPG Photonics GmbH, Burbach, Germany) was aligned with the optical axis of the microscope and directed into the objective lens to form the trap in its focus. Powers between 10 to $20 \mathrm{~mW}$ at the sample were used to trap individual liposomes. After selection in bright field imaging, a single liposome was trapped. With the liposome fixed in the trap, the stage was moved laterally to position the liposome at the desired site [Fig. 1(a)]. The vertical position could also be adjusted moving the objective. To achieve optimum trapping at long working distances $(\mathrm{WD})$, the objective lens $(100 \times, \mathrm{NA}=1, \mathrm{WD}=1)$ was chosen. This allowed the trapping of the liposome inside the capillary and the ability to keep it fixed in the trap during transportation outside. The release of liposome content was induced by breaking the vesicle membrane with short UV laser pulses (wavelength $355 \mathrm{~nm}$, energy $<1 \mu \mathrm{J} /$ pulse, pulse duration $<0.5 \mathrm{~ns}$, repetition rate $<5 \mathrm{kHz}$ ), using a microdissection system (MMI-CellCut Plus, MMI, Zurich, Switzerland) implemented on the same microscope. After liposome rupture, the two lasers were switched off and the sample was monitored in epi-fluorescence mode.

$\mathrm{Ca}^{2+}$ imaging experiments were performed in epifluorescence using a blue excitation filter block (B-2A, Nikon). The fluorescence images were recorded with a CCD camera DVC-1412AM (DVC Company, Austin, Texas). Frames $(174 \times 130$ pixels in $8 \times 8$ binning mode $)$ were collected at the rate of $22 \mathrm{~Hz}$ at 12-bits. Stock solution of the calcium dye Oregon Green 488 BAPTA-1-AM (OGB-1-AM, Molecular Probes O-6807) was prepared in dimethyl sulfoxide. Cell cultures were then incubated for $30 \mathrm{~min}$ with $1.5 \mu \mathrm{M}$ of the $\mathrm{Ca}^{2+}$ dye. After incubation, neuronal cultures were washed and transferred on the stage of the microscope. Measurements were performed at $37^{\circ} \mathrm{C}$. Data were analyzed with software ImageJ and Matlab (Mathworks, Natick, Massachusetts). Increases of $\mathrm{Ca}^{2+}$ are expressed in relative fluorescence changes (DF/F) as described in Ref. 15.

Liposomes with dimension ranging from 1 to $10 \mu \mathrm{m}$ were produced as previously described. The efficiency of encapsulation and subsequent release induced by the UV pulse, was first qualitatively evaluated [Figs. 1(b) and 1(c)].

Liposomes were encapsulated with $1 \mathrm{mM}$ fluorescein and collected in a reservoir consisting of a rectangular glass capillary. The reservoir was introduced in the recording chamber and a single liposome was optically trapped, positioned, and visualized both in bright field and epi-fluorescence [Fig. 1(b)]. The fluorescence signal originated from the liposome allowed us to check the correct encapsulation [Fig. 1(b), left]. After UV-pulse delivery, fluorescence disappeared from the liposome demonstrating complete depletion of fluorescein and a shape rearrangement of the vesicle induced by the microdissection occurred [Fig. 1(b), right]. The fluorescence signal from a large vesicle in close vicinity was not influenced by the UV pulse [Fig. 1(b)], demonstrating single vesicle photolysis.

For mono/oligo-lamellar vesicles of 1 to $10 \mu \mathrm{m}$, i.e., the range used in our experiments, the efficiency of triggering release was $100 \%$, while multi-lamellar vesicles often required separated and repeated UV trains. 
(a)
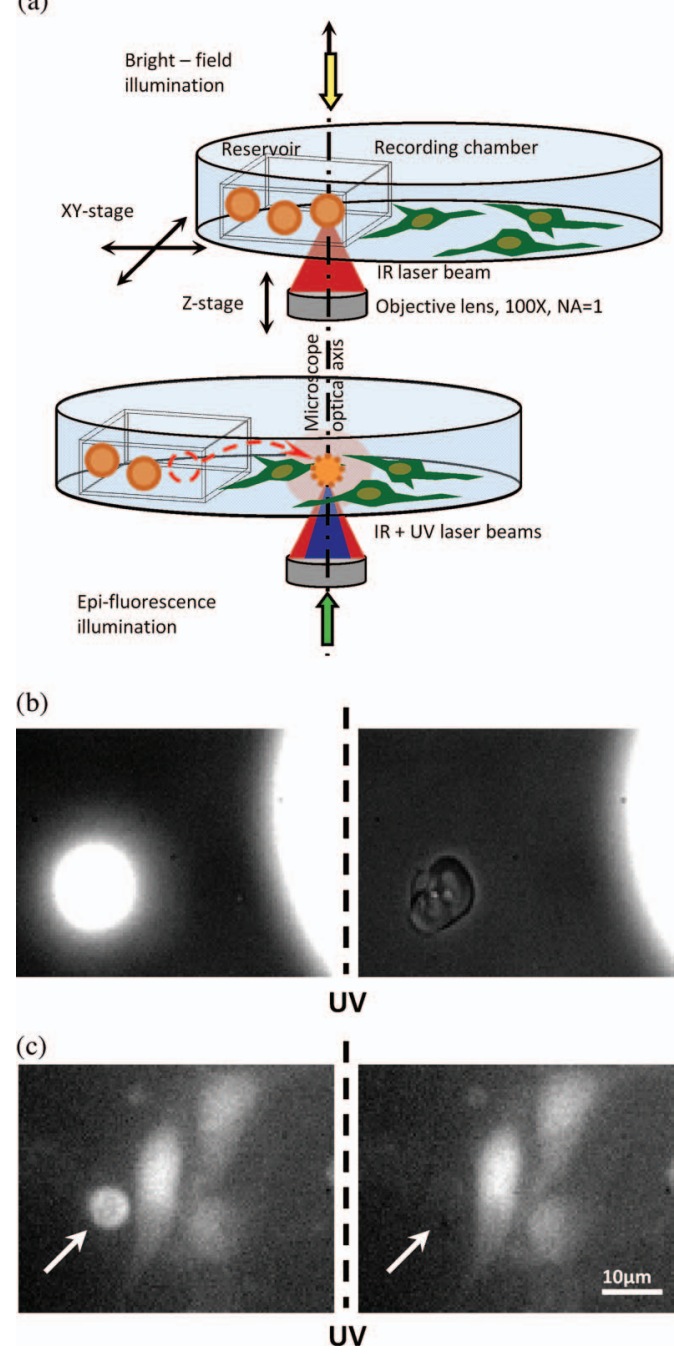

Fig. 1 Optical manipulation and dissection of a single liposome and characterization of the efficiency of encapsulation and release. (a) A selected liposome inside the capillary is first fixed in the optical trap (top) and then placed to the specific site near the cells by moving the stage and microscope objective (black arrows). A short train of UV pulses breaks the liposome to deliver the content to the surrounding environment (bottom). (b) Simultaneous bright field and fluorescence images before (left) and after (right) UV-pulse release from a liposome containing $1 \mathrm{mM}$ fluorescein. (c) A fluorescent liposome was optically trapped and brought in the vicinity of cultured neuronal cells treated with OGB-1-AM. Fluorescence was measured before and after the UVinduced release, allowing to test the efficiency of encapsulation and subsequent release.

Liposome content could be released in the proximity of neurons without damage. As shown in Fig. 1(c), the fluorescent content of a single liposome positioned close to a group of neuronal cells stained with OGB-1-AM, was released. The fluorescence signal from the vesicle disappeared, revealing complete release of the liposome content, while the baseline fluorescence signal from the $\mathrm{Ca}^{2+}$ dye stained neurons was still present.

Subsequently, the effect of release from liposomes was tested on neuronal cells in culture [Fig. 2(a)], by releasing encapsulated $\mathrm{KCl}$. Neuronal responses were monitored with a $\mathrm{Ca}^{2}+$ imaging technique, by loading the cells with OGB-1-AM. From the baseline fluorescence (not shown) it was possible to identify the contribution of single neurons and associate them (a)
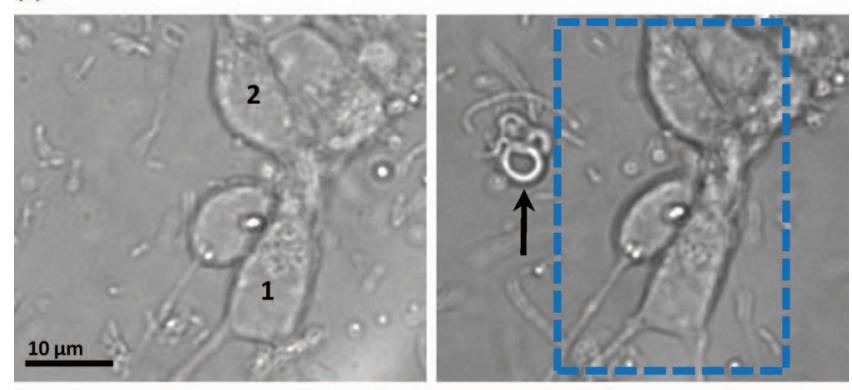

(b)
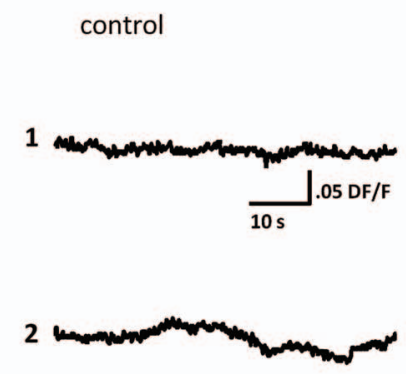

(c)
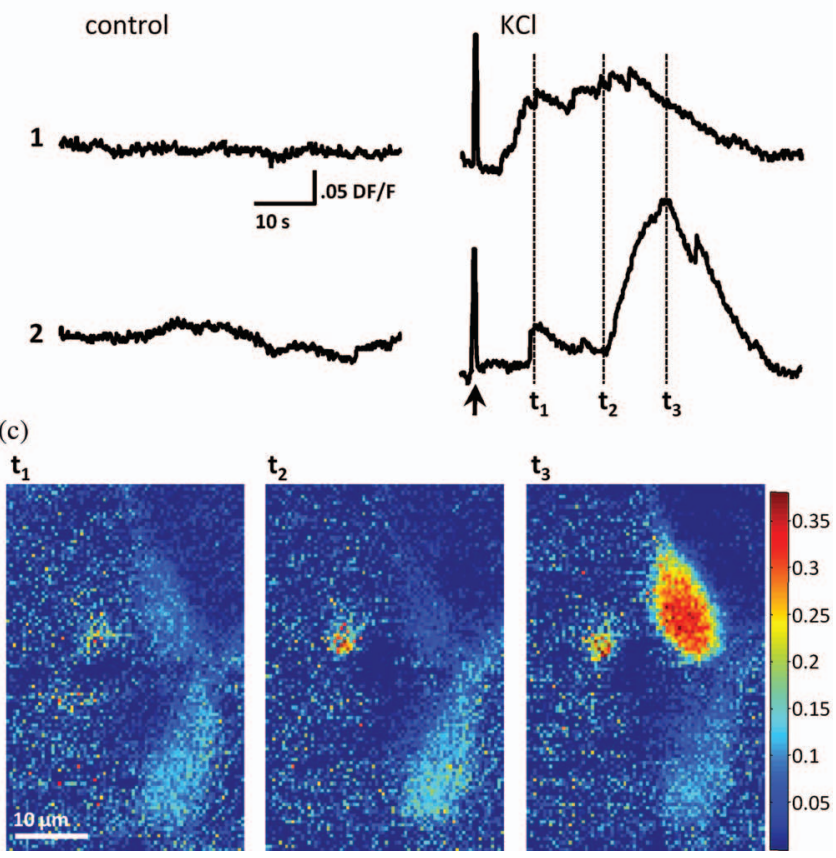

Fig. 2 Optical delivery of high $\mathrm{KCl}$ to neurons and simultaneous measurements of $\mathrm{Ca}^{2}+$ elevation responses by fluorescence imaging. (a) Bright field images of cultured hippocampal neurons before (left) and after (right) the positioning of a single liposome (black arrow) encapsulated with KCl $100 \mathrm{mM}$. (b) Time dependence of DF/F, calculated from cells 1 and 2 as indicated in (a). Left traces report spontaneous fluctuations of $\mathrm{Ca}^{2}+$ fluorescence in absence of external stimuli (control), while right traces were recorded after UV-pulse induced $\mathrm{KCl}$ release $(\mathrm{KCl})$. The presence of an artifact (arrow) indicates the time of release. (c) Three images reporting the values of DF/F expressed in pseudo-colors, over the area indicated in the box of panel (a) for the time points $t_{1}, t_{2}$, and $t_{3}$ as indicated in (b).

to different regions of interest. The activity was monitored by calculating the value of $\mathrm{DF} / \mathrm{F}$ over time. Cells were first monitored for a few minutes before the stimulation in order to obtain a control on the spontaneous activity [Fig. 2(b), left traces, numbers 1 and 2 refers to the cells indicated in Fig. 2(a), left]. After this period, a single liposome with a suitable volume was selected in the reservoir by visual inspection. The liposome could be trapped with the optical tweezers, and by moving the microscope stage it could be positioned at the location of interest as indicated by the arrow in Fig. 2(a). The liposome was then cut by UV dissection while monitoring fluorescence signals from the neuronal culture. In order to avoid any disturbance on the measurements of fluorescence emission from the $\mathrm{Ca}^{2+}$ dye, fluorescein was not included in these experiments. Following 
(a)
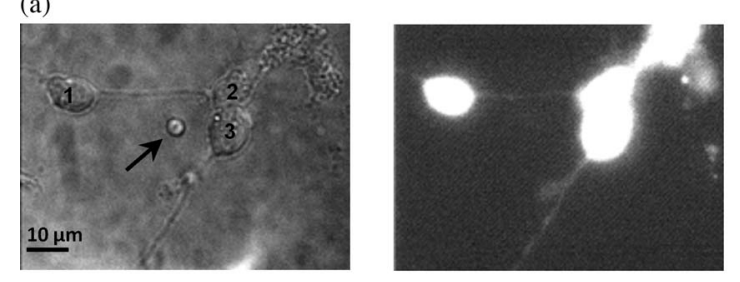

(b)

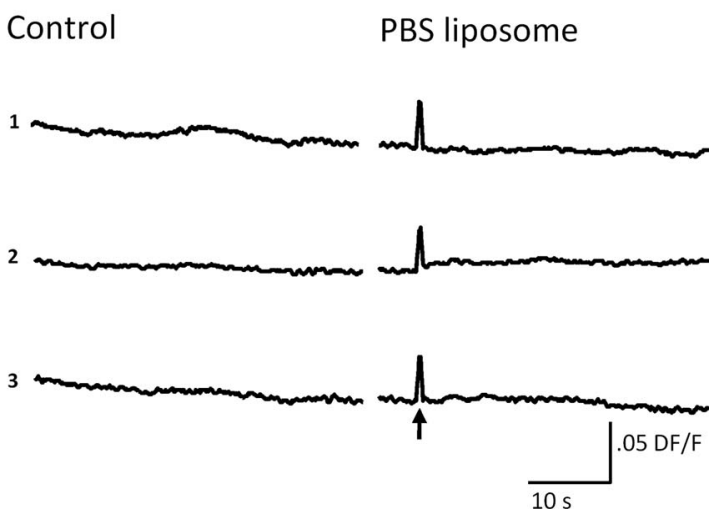

Fig. 3 Photolysis of control liposomes containing PBS. (a) Bright field (left) and fluorescence image (right) of a neuronal culture treated with a control liposome containing PBS. (b) Time dependence of DF/F, calculated from cells 1, 2, and 3 as indicated in (a). Left traces report spontaneous fluctuations of $\mathrm{Ca}^{2+}$ fluorescence in absence of external stimuli, while right traces were recorded after UV-laser photolysis of a control liposome [(a), arrow]. The artifacts indicate the time of release (arrow).

the dissection of the liposome the time dependence of DF/F mediated over the different cells showed a large increase, followed by a return on the baseline level [Fig. 2(b), right] denoting a depolarization induced $\mathrm{Ca}^{2}+$ influx originated by the release of $\mathrm{KCl}$. DF/F images [Fig. 2(c)] were also calculated at different time points $\left[t_{1}, t_{2}, t_{3}\right.$, indicated in Fig. 2(b), right]. These data show the distribution of activation within the area corresponding to the neuronal cells. Differences in cell responses [Figs. 2(b) and 2(c)] can be ascribed to the heterogeneity of the intrinsic electrical properties of the neurons present in the cultures and to the presence of both inhibitory and excitatory synaptic connections that influence the electrical activity. Altogether, these results confirm the possibility of focal delivering chemical stimuli to neuronal cells through release from liposomes. In order to exclude artifacts from the UV treatment of the liposomes, control experiments were done by delivering PBS from liposomes. No response was observed after the PBS release, induced by UV pulse (Fig. 3). This control also excluded the possibility of any effect of the specific lipid composition of the liposomes on the neurons, as membrane-distorting effects.

Intracellular drug delivery through liposomes is a widely diffuse technique that exploits the fusion of liposomes in the cellular membrane. ${ }^{13,16-19}$ Here, alternatively, using micrometric vesicles as vectors, we extended a technique previously introduced by Sun et al. ${ }^{9}$ to deliver extracellular chemical stimuli to morphologically complex cells as neurons are. We demonstrate the possibility of local stimulation of hippocampal primary neuronal cultures, a model system composed of cells of complex morphology, synaptically connected in a network. We also show that neuronal electrical activity can be evoked with this technique, which opens new opportunities for the electrophysiological investigation of neuronal receptors and ion channels.

A main advantage of the method is the possibility to accurately measure the volume of the liposome, that ranges from femtoliters to picoliters for liposomes ranging from 1 to $10 \mu \mathrm{m}$, and consequently, to estimate the amount of molecules released, given the concentration. The time precision of delivery is sub$\mathrm{ms}$, although, the distance from the delivery point must be taken into account to provide a correction of both the actual concentration and precise occurrence of the stimulus. ${ }^{8}$

Another advantage consists in the procedure of liposome production and encapsulation that is very simple and does not require expensive equipment. Moreover, the amount of drug solution that is necessary for the encapsulation procedure can be kept very modest, allowing to perform experiments on a large number of neurons with reduced cost for pharmacological agents. Finally, since the procedure allows only local chemical stimulation, several experiments can be performed on the same chamber, allowing performance of experiments with reduced sample to sample variations and limited animal sacrifice.

\section{Acknowledgments}

We thank Barbara Sartori and Michael Rappolt for help in the preparation of the liposomes and for useful discussions. Sources of support: EU project Nanoscale 214566.

\section{References}

1. J. E. Corrie, A. DeSantis, Y. Katayama, K. Khodakhah, J. B. Messenger, D. C. Ogden, and D. R. Trentham, "Postsynaptic activation at the squid giant synapse by photolytic release of L-glutamate from a 'caged' L-glutamate," J. Physiol. (London) 465, 1-8 (1993).

2. A. G. Pakhomov, I. Semenov, R. Brenner, and G. M. Toney, "Hydraulically coupled microejection technique for precise local solution delivery in tissues," J. Neurosci. Methods 155(2), 231-240 (2006).

3. V. I. Pidoplichko and J. A. Dani, "Applying small quantities of multiple compounds to defined locations of in vitro brain slices," J. Neurosci. Methods 142(1), 55-66 (2005).

4. A. Losonczy and J. C. Magee, "Integrative properties of radial oblique dendrites in hippocampal CA1 pyramidal neurons," Neuron 50(2), 291307 (2006).

5. G. C. Ellis-Davies, "Caged compounds: photorelease technology for control of cellular chemistry and physiology," Nat. Methods 4(8), 619628 (2007).

6. M. A. Smith, G. C. Ellis-Davies, and J. C. Magee, "Mechanism of the distance-dependent scaling of Schaffer collateral synapses in rat CA1 pyramidal neurons," J. Physiol. (London) 548(Pt 1), 245-258 (2003).

7. M. Canepari, L. Nelson, G. Papageorgiou, J. E. Corrie, and D. Ogden, "Photochemical and pharmacological evaluation of 7-nitroindolinyland 4-methoxy-7-nitroindolinyl-amino acids as novel, fast caged neurotransmitters," J. Neurosci. Methods 112(1), 29-42 (2001).

8. H. Kress, J.-G. Park, C. O. Mejean, J. D. Forster, J. Park, S. S. Walse, Y. Zhang, D. Wu, O. D. Weiner, T. M. Fahmy, and E. R. Dufresne, "Cell stimulation with optically manipulated microsources," Nat. Methods 6(12), 905-909 (2009).

9. B. Sun and D. T. Chiu, "Spatially and temporally resolved delivery of stimuli to single cells," J. Am. Chem. Soc. 125(13), 3702-3703 (2003).

10. H. H. Hub, U. Zimmermann, and H. Ringsdorf, "Preparation of large unilamellar vesicles," FEBS Lett. 140(2), 254-256 (1982).

11. M. Ichikawa and K. Yoshikawa, "Optical transport of a single cell-sized liposome," Appl. Phys. Lett. 79(27), 4598-4600 (2001).

12. J. M. Berg, J. L. Tymoczko, and L. Stryer, Biochemistry, Freeman, San Francisco (2002). 
13. R. Rahamimoff, H. Meiri, S. D. Erulkar, and Y. Barenholz, "Changes in transmitter release induced by ion-containing liposomes," Proc. Natl. Acad. Sci. U.S.A. 75(10), 5214-5216 (1978).

14. B. Sun and D. T. Chiu, "Determination of the encapsulation efficiency of individual vesicles using single-vesicle photolysis and confocal singlemolecule detection," Anal. Chem. 77(9), 2770-2776 (2005).

15. G. Pinato, S. Pegoraro, G. Iacono, M. E. Ruaro, and V. Torre, "Calcium control of gene regulation in rat hippocampal neuronal cultures," J. Cell. Physiol. 220(3), 727-747 (2009).

16. K. Tsumoto, S.-I. M. Nomura, Y. Nakatani, and K. Yoshikawa, "Giant liposome as a biochemical reactor: transcription of DNA and transportation by laser tweezers," Langmuir 17(23), 7225-7228 (2001).
17. M. J. Ostro and P. R. Cullis, "Use of liposomes as injectabledrug delivery systems," Am. J. Hosp. Pharm. 46(8), 1576-1587 (1989).

18. M. Kaneda, S.-I. M. Nomura, S. Ichinose, S. Kondo, K.-i. Nakahama, $\mathrm{K}$. Akiyoshi, and I. Morita, "Direct formation of proteo-liposomes by in vitro synthesis and cellular cytosolic delivery with connexin-expressing liposomes," Biomaterials 30(23-24), 3971-3977 (2009).

19. A. Stromberg, F. Ryttsen, D. T. Chiu, M. Davidson, P. S. Eriksson, C. F. Wilson, O. Orwar, and R. N. Zare, "Manipulating the genetic identity and biochemical surface properties of individual cells with electric-field-induced fusion," Proc. Natl. Acad. Sci. U.S.A. 97(1), 7-11 (2000). 